\title{
Effects of remote digital monitoring on oral hygiene of orthodontic patients: a prospective study
}

\author{
Linda Sangalli ${ }^{1,2}$, Fabio Savoldi ${ }^{3}$ (D), Domenico Dalessandri ${ }^{4}$, Stefano Bonetti ${ }^{4}$, Min Gu$^{3^{*}} \mathbb{D}$, \\ Alberto Signoroni ${ }^{1}$ and Corrado Paganelli ${ }^{4}$
}

\begin{abstract}
Background: Remote digital monitoring during orthodontic treatment can help patients in improving their oral hygiene performance and reducing the number of appointments due to emergency reasons, especially in time of COVID-19 pandemic where non-urgent appointments might be discouraged.

Methods: Thirty patients scheduled to start an orthodontic treatment were divided into two groups of fifteen. Compared to controls, study group patients were provided with scan box and cheek retractor (Dental Monitoring ${ }^{\circledR}$ ) and were instructed to take monthly intra-oral scans. Plaque Index (PI), Gingival Index (GI), and White Spot Lesions (WSL) were recorded for both groups at baseline $\left(t_{0}\right)$, every month for the first 3 months $\left(t_{1}, t_{2}, t_{3}\right)$, and at 6 months $\left(t_{4}\right)$. Carious Lesions Onset (CLO) and Emergency Appointments (EA) were also recorded during the observation period. Inter-group differences were assessed with Student's $t$ test and Chi-square test, intra-group differences were assessed with Cochran's Q-test (significance $a=0.05$ ).

Results: Study group patients showed a significant improvement in plaque control at $t_{3}(p=0.010)$ and $t_{4}(p=0.039)$, compared to control group. No significant difference was observed in the number of WSL between the two groups. No cavities were detected in the study group, while five CLO were diagnosed in the control group $(p=0.049)$. A decreased number of EA was observed in the study group, but the difference was not significant.

Conclusions: Integration of a remote monitoring system during orthodontic treatment was effective in improving plaque control and reducing carious lesions onset. The present findings encourage orthodontists to consider this technology to help maintaining optimal oral health of patients, especially in times of health emergency crisis.
\end{abstract}

Keywords: Oral hygiene, Telemonitoring, Dental Monitoring, Orthodontics, Digital dentistry, COVID-19

\section{Background}

Oral hygiene should be routinely controlled in patients undergoing orthodontic treatment. Several studies have demonstrated a rapid decline in the level of oral hygiene status after the initial bonding of the orthodontic

\footnotetext{
*Correspondence: drgumin@hku.hk

${ }^{3}$ Division of Paediatric Dentistry and Orthodontics, Faculty of Dentistry,

2/F, Prince Philip Dental Hospital, The University of Hong Kong, 34

Hospital Road, Sai Ying Pun, Hong Kong SAR

Full list of author information is available at the end of the article
}

appliance [1], which constitutes an obstacle to the oral hygiene procedures [2] and may lead to changes in composition of the bacterial flora [3]. Presence of dental plaque on the tooth surface for a critical length of time is associated with increased chance of demineralization and white spot lesions [4], along with gingival inflammation [4]. This can negatively affect the clinical outcome due to possible discontinuation of the orthodontic treatment [4]. Moreover, teenagers may be at high risk of carious 
lesion onset because of lack of cooperation and difficulties during the daily oral hygiene procedures [5].

Nevertheless, oral hygiene status can significantly ameliorate with reward systems or active reminder tools, especially when teenagers are engaged by technological supports [6,7]. Two systematic reviews have shown a positive influence of text messages on behavioural changes [8], and a significant association between the use of mobile technologies and the improvement in dental plaque control and gingival bleeding [9]. Previous studies proposed a system of active reminders for adolescents undergoing orthodontic treatment by weekly text messaging their parents $[8,10-12]$, using WhatsApp chat room for sharing "selfies" of their smile [13], and using computer-based training to teach Fones brushing technique or modified Bass technique [14]. In addition, frequently used social media among young subjects, such as Instagram [15], or other digital platforms, such as YouTube [16], have shown to improve oral health knowledge among orthodontic patients. Still, some studies found no positive effect of instructions about oral hygiene during orthodontic treatment by using social media-based and messaging apps, respectively $[17,18]$.

A recent new tool for remote monitoring is Dental Monitoring ${ }^{\circledR}$ (DM, Paris, France), a software-based program that allows patients to capture their occlusion using a smart phone and a scan box. It consists of three integrated platforms: a mobile app for the user, a movementtracking algorithm, and a web-based Doctor Dashboard ${ }^{\circledR}$, where the clinician can check the treatment progress, teeth movement, integrity of appliances, and oral hygiene status through the analysis of pictures that are periodically taken by the patient [19]. Such remote monitoring is especially important in times of COVID-19 pandemic, as it allows maintaining continuity of care, while minimizing the risk of disease transmission and optimizing the use of resources [20].

To the best of our knowledge, the present work may be the first investigation of the oral hygiene status of orthodontic patients using the scan box. The aim of the study was to verify whether an active reminder-such as DMintegrated to the traditional orthodontic standard of care, could help patients in maintaining a better oral hygiene during the first six months of treatment, and in reducing the number of appointments due to emergency reasons.

\section{Methods}

\section{Study subjects}

Setting a clinically significant difference of 0.5 points in the Plaque Index (PI) between the two groups, a Standard Deviation (SD) of 0.5 based on a previous study [21], a significance level $\alpha=0.05$, and a power beta $=80 \%$, the required sample size was calculated as 17 subjects for each group [22]. Considering the drop-out rate, forty consecutive patients scheduled to start an orthodontic treatment between January and December 2018 were proposed to participate in the study. Eight of them deviated from the inclusion criteria during the study and two eventually declined to participate. Inclusion criteria were to be online daily, to have access to a smartphone, and to undergo a non-extraction orthodontic treatment with fixed brackets or aligners. Exclusion criteria were a daily supplemental fluoride regimen, and physical or cognitive disabilities impeding to take pictures or to perform oral hygiene procedures.

Among the thirty participants enrolled in the study (mean age 20.6 \pm 9.0 years), 15 patients ( 7 males and 8 females, mean age $24.9 \pm 10.9$ years) were assigned to the study group: 5 patients were treated with fixed buccal multi-bracket appliance, and 10 patients with aligners. The control group (15 patients, 7 males and 8 females, mean age $16.3 \pm 3.2$ years) consisted of 11 patients treated with fixed buccal multi-bracket appliance, 3 patients with aligners, and 1 patient with fixed lingual multi-bracket appliance.

The study was performed in accordance with the ethical standards as laid down in the Declaration of Helsinki and was approved by the Institutional Review Boards of the University of Brescia.

\section{Treatment protocol}

Both groups were treated by the same orthodontist (L.S.). The fixed buccal multi-brackets were self-ligating Empower ${ }^{\circledR}$ brackets with MBT prescription and 0.022inch slot (American Orthodontics, Sheboygan, WI), applied with direct bonding technique. Patients undergoing treatment with aligners received Invisalign ${ }^{\circledR}$ appliances (Align Technology, San Jose, CA). The fixed lingual multi-bracket appliance was Win ${ }^{\circledR}$ (DW Lingual Systems, Bad Essen, Germany), applied with indirect bonding technique.

\section{Oral hygiene protocol and assessment}

An oral hygiene kit, containing toothbrush and toothpaste (Mentadent ${ }^{\circledR}$, Unilever, the Netherlands), a mouthwash and a dental floss $\left(\mathrm{GUM}^{\circledR}\right.$, Sunstar Suisse, Switzerland), and an inter-proximal aid (Krugg, Melville, New York, USA) were provided to each patient, with the instructions to brush their teeth at least twice per day and floss once per day. The patients and their parents were educated by the clinician during the first in-person visit, also regarding the importance of limiting sugar consumption and avoiding sticky food.

During the appointment of the bonding of the appliance or of the delivery of the aligners $\left(\mathrm{t}_{0}\right)$, every month for the first three months $\left(t_{1}, t_{2}, t_{3}\right)$ and at 6 months $\left(t_{4}\right)$, 
Plaque Index (PI), Gingival Index (GI), and White Spot Lesions (WSL) were clinically assessed [23].

At chair-side visits, PI was scored by evaluating the presence of plaque on the mesial, buccal, distal and lingual surfaces of 1.6, 1.4, 2.1, 4.6, 3.2, 3.5, assigning a score from 0 to 3 for each surface and calculating the mean value (Table 1) [24]. GI was scored by evaluating the presence of inflammation on the buccal surfaces of 1.6, $1.4,2.1,4.6,3.2,3.5$, assigning a score from 0 to 3 and calculating the mean value (Table 1) [24]. To evaluate WSL, teeth were air-dried for $5 \mathrm{~s}$ and then the buccal surface close to the gingival contour was evaluated, assigning a score from 0 to 3 (Table 1). [24].

For both groups, the number of Emergency Appointment (EA) and Carious Lesions Onset (CLO) were recorded during the observation period. At each visit, both groups were additionally monitored using plaquedisclosing tablets (Red-Cote ${ }^{\circledR}$, GUM, Sunstar Suisse, Switzerland). The outcome was photographed and shown to the patient.

In addition to the chair-side appointments, the study group was also monitored with remote $2 \mathrm{D}$ photo monitoring (Dental Monitoring ${ }^{\circledR}$, DM, Paris, France) (Fig. 1). At baseline, the study group patients were asked to download the DM app, and were instructed to take pictures of their mouth to be uploaded. A scan box and a dedicated cheek retractor by DM were provided to each patient of the study group. The first scan was made together with the orthodontist to ensure proper use of the device. The frequency of scans was monthly, and DM evaluated the pictures uploaded by the patient upon the oral hygiene status, checking the periodontal gingival health, the

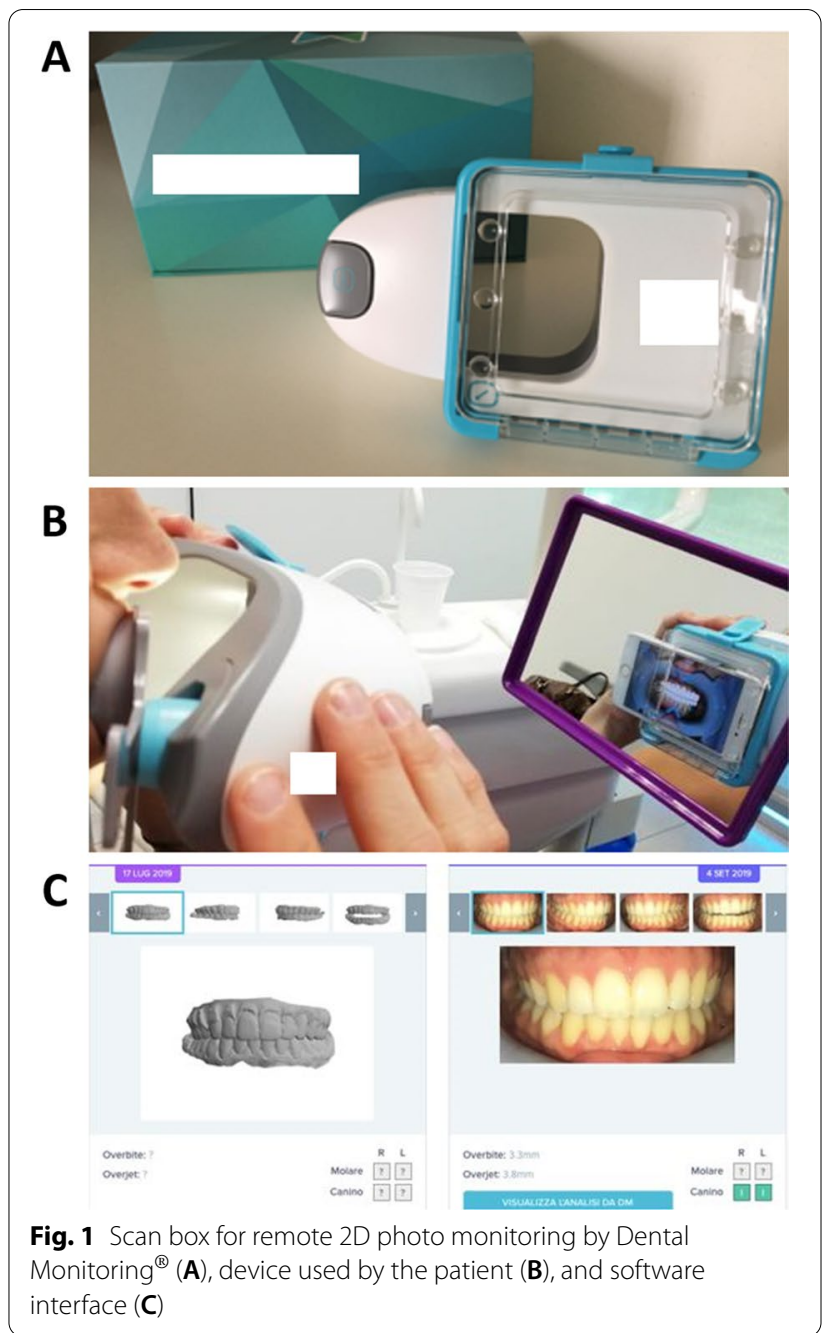

Table 1 Plaque Index, Gingival Index and White Spot Lesions scales used for clinical evaluation

\begin{tabular}{ll}
\hline Plaque Index (PI) score & \\
0 & Absence of plaque in the gingival area \\
1 & Slight deposit of plaque at gingival margin \\
2 & Moderate accumulation of soft deposits covering less than half of the surface \\
3 & Abundance of deposits covering more than half of the surface \\
Gingival Index (GI) score & \\
0 & Normal gingiva, no inflammation, bleeding or swelling \\
1 & Mild inflammation, slight edema and color change; no bleeding \\
2 & Moderate inflammation, redness, swelling; bleeding when probing \\
3 & Important inflammation, marked redness and edema; spontaneous bleeding \\
White Spot Lesions (WSL) score & \\
0 & No visible WSL or surface disruption (no demineralization) \\
1 & Visible WSL without enamel surface disruption (mild demineralization) \\
2 & Visible WSL with roughened surface (moderate demineralization) \\
3 & Visible WSL requiring restoration (severe demineralization)
\end{tabular}




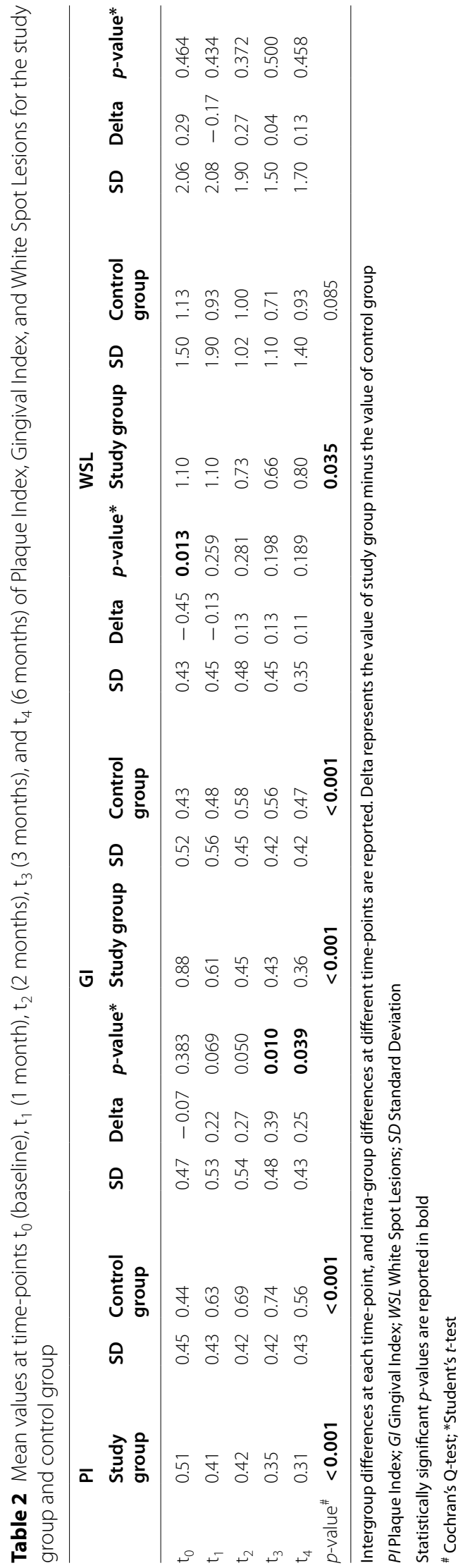


amount of plaque left on the teeth, and sent a text message to the patient with its evaluation.

\section{Data analysis}

The normality of the data distribution was verified with Shapiro-Wilk test. Differences between groups regarding PI, GI, and WSL were assessed by Student's $t$-test. Intragroup differences of PI, GI, and WSL at different timepoints were assessed using Cochran's Q-test. Chi-Square test was used to compare CLO and EA between the two groups. Statistical analysis was performed with statistical software $\left(\mathrm{SPSS}^{\odot}\right.$ Statistics 27, IBM, Armonk, NY, USA) at significance level $\alpha=0.05$.

\section{Results}

\section{Intra-group differences}

The intra-group differences at different time-points for study group patients were significant for PI, GI and WSL (Table 2). PI values decreased from $0.51( \pm 0.45)$ at $t_{0}$, to $0.31( \pm 0.43)$ at $\mathrm{t}_{4}(p<0.001)$. GI values decreased from $0.88( \pm 0.52)$ at $\mathrm{t}_{0}$, to $0.36( \pm 0.42)$ at $\mathrm{t}_{4}(p<0.001)$. WSL values decreased from $1.10( \pm 1.50)$ at $t_{0}$, to $0.80( \pm 1.40)$ at $\mathrm{t}_{4}(p=0.035)$.

In the control group, PI and GI values increased significantly, while WSL values did not reveal a significant change (Table 2). PI values increased from $0.44( \pm 0.47)$ at $\mathrm{t}_{0}$, to $0.56( \pm 0.43)$ at $\mathrm{t}_{4}(p<0.001)$. GI values increased from $0.43( \pm 0.43)$ at $\mathrm{t}_{0}$, to $0.47( \pm 0.35)$ at $\mathrm{t}_{4}(p<0.001)$.

\section{Inter-group differences}

Regarding PI, despite the two groups had similar values at baseline, at $t_{3}$ the mean value of the study group was significantly lower $(0.35 \pm 0.42)$ compared to the control group $(0.74 \pm 0.48)(p=0.010)$. At $\mathrm{t}_{4}$, the mean value of the study group was also significantly lower $(0.31 \pm 0.43)$ compared to the control group $(0.56 \pm 0.43)(p=0.039)$ (Table 2 and Fig. 2).

With regard to GI, even though at baseline the mean value for the study group was higher $(0.88 \pm 0.52)$ compared to the control group $(0.43 \pm 0.43)(p=0.013)$, the two groups reached similar values during the observation period (Table 2 and Fig. 2).

The difference between the two groups with regard to WSL was not significant at any timepoint (Table 2 and Fig. 2).

As for CLO, no cavities were detected in the study group, while five CLO were diagnosed in the control group $(p=0.049)$.

With regard to EA, although the patients of the control group recorded more extra appointments (7.0) than those of the study group (3.9), the difference was not statistically significant.

\section{Discussion}

Systems of tele monitoring have become one of the most widespread response of the medical field to the current COVID-19 pandemic [25] and-in appropriate caseshave shown to be as effective and well-accepted by patients as the standard care of treatment [26]. Remote monitoring systems are part of Artificial Intelligence Driven Remote Monitoring (AIRM) [27]. Several clinical applications of these technologies in orthodontics include monitoring the integrity and side effects of the appliance, the gingival health of the patients, and the loss of tracking of the dental movements obtained with aligners [28].

The present study focused on the monitoring of orthodontic patients, and the record of PI, originally described by Silness and Loe [24], was one of the parameters selected to evaluate the level of their oral hygiene status. In the literature, the PI was used in the majority of the trials [29], as it allows a rapid assessment and it is workable in dental offices without expensive costs. According to best clinical practice principles, plaque assessment further included the use of disclosingplaque tablets, which evidence was photographed and shown to patients and parents in order to enhance collaboration and oral hygiene independently from the use of remote digital monitoring. In fact, it is an affordable and easy-to-perform visual method that provides a rapid feedback to improve brushing technique and conscious awareness [30]. With regard to PI, the control group showed a worsening of the oral hygiene level, although after three months the accumulation of plaque substantially decreased. This might be interpreted as a sign that instructions and visual method of disclosing-plaque tablets eventually enhanced awareness in patients and families on the importance of a good oral hygiene [31]. Yet, the final PI value at six months remained higher than the value at baseline, confirming the difficulty of patients in keeping teeth clean during orthodontic treatment [32], which may be especially difficult in case of fixed appliances with complex design [33]. As for the study group, PI values steadily decreased over time, and after six months the plaque detected was less than the initial value. Overall, the trend shown by the two groups was the opposite, in accordance with the literature [34]. Accordingly, the differences between control group and study group regarding PI were significant during the latest assessments at three and six months.

With regard to GI, the control group showed a worsening of the periodontal health status during the first months of treatment, according to the literature [30]. Conversely, in the study group, the GI dropped by more than half of the initial values by the end of the observation period. However, the difference between the two 


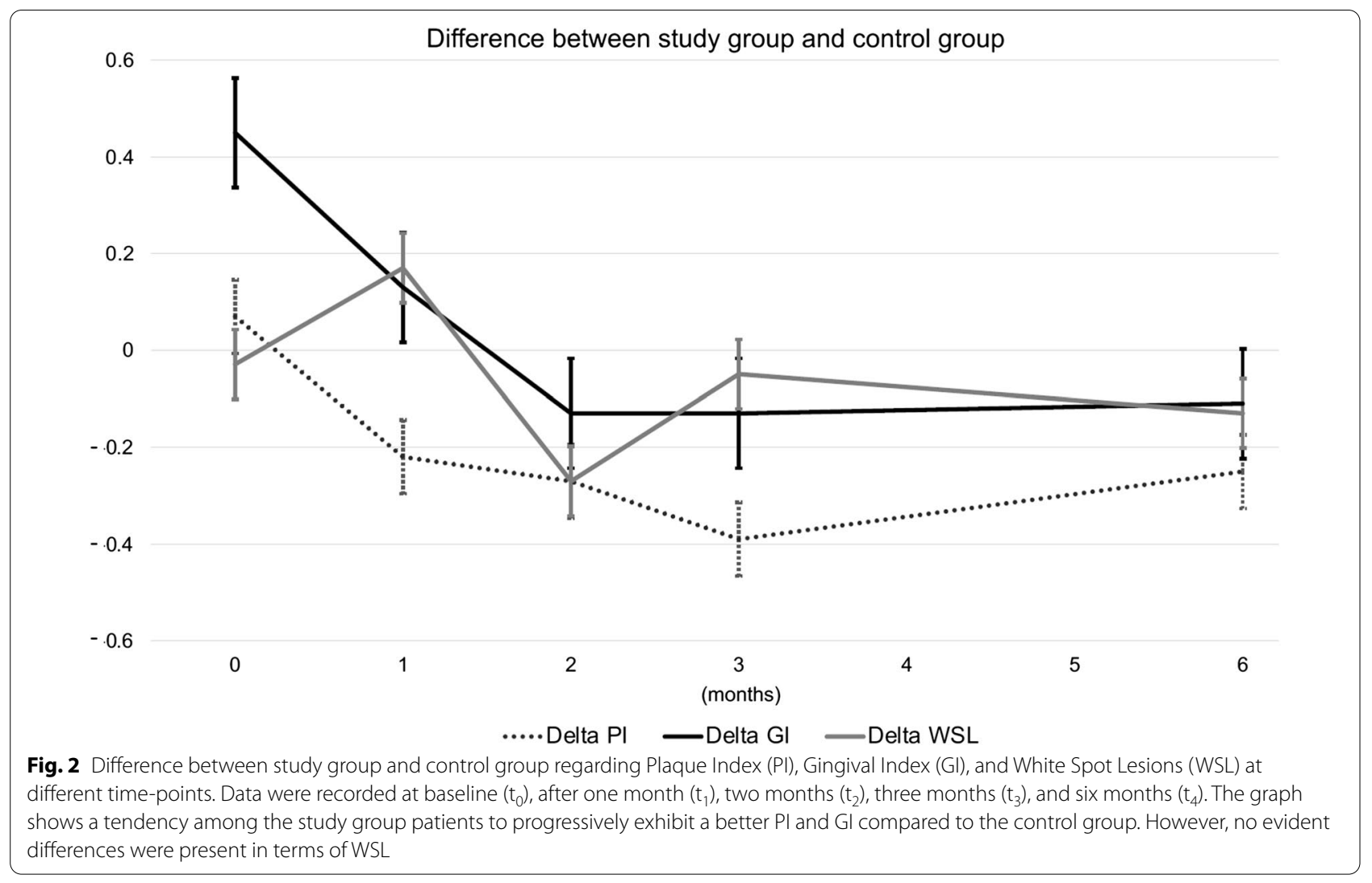

groups was not significant and it is unclear whether DM also helped to improve the periodontal status.

Concerning WSL, their onset steadily decreased over time in the study group. It might be possible that implements aimed to improve oral hygiene also helped in increasing cooperation of patients, in accordance with a systematic review [31]. Still, the difference between control group and study group was not significant and the present study was inconclusive in showing improvements of this aspect when DM was used.

A relevant finding was the difference in terms of CLO between the two groups, where DM is likely to have played an important role in enhancing the attention of the patients on oral hygiene control. Still, the overall improvement in oral hygiene of the study group might be partly due to the Hawthorne Effect [35], as patients in the study group were aware of being under monitoring by the examiner.

According to the literature, monitoring the oral hygiene status of orthodontic patients may decrease the number of EA [36, 37]. The present study confirmed a similar trend, even though the reduction of extra appointments in the study group was not statistically significant.

Nevertheless, such remote digital technologies may include potential concerns, including a possible deterioration in patient-clinician relationship due to a reduced number of in-person appointments, and the inevitable cost of using AIRM itself [28]. Considering the novelty of such technological advances and the lack of well-defined standards [27], the clinicians should carefully balance the benefits of in-office visits with the advantages of remote monitoring, while maintaining standard of care.

\section{Limitations}

The value of GI was significantly different at baseline between study group and control group, and randomized studies are necessary to confirm the present findings. Further works may also extend the observation period to one year, in order to complete the orthodontic treatment, or longer, to observe the retention period as well. In the present study, patients used DM dedicated cheek retractors when taking the scans. However, every person is unique in the amount of maximal mouth opening and cheek muscle tonicity, which may have affected the tooth visibility in the oral cavity. Moreover, the scans of two 10-year-old patients were often rejected by the software, maybe due to the poor manual skills of such young subjects. Thus, how well teeth are captured may vary 
depending on the manual skills of each individual, and such variation may have influenced the present results.

\section{Conclusions}

Remote monitoring applied during orthodontic treatment showed encouraging results in reducing plaque and onset of carious lesions. However, incidence of emergency appointments, gingival status, and onset of white spot lesions may not significantly improve. These preliminary results suggest potential application of this technology in clinical practice, especially in times when routine clinical check-ups might be compromised. Further randomised studies including larger and more homogeneous groups of participants are advisable to confirm the present findings.

\section{Abbreviations}

DM: Dental Monitoring; PI: Plaque Index; GI: Gingival Index; WSL: White Spot Lesions; CLO: Carious Lesion Onset; EA: Emergency Appointments; COVID19: Coronavirus disease 2019; AIDRM: Artificial intelligence driven remote monitoring.

\section{Acknowledgements \\ Not applicable.}

\section{Authors' contributions}

LS collected the patients data; FS and LS analyzed and interpreted the data; DD developed the study protocol; LS and FS contributed to the drafting and writing of the manuscript. DD, MG, CP, SB, and AS contributed with expertise advice and critically revised the manuscript. All authors read and approved the final manuscript.

\section{Funding}

Dental Monitoring ${ }^{\circledR}$ partially sponsored the cost of the monthly monitoring.

\section{Availability of data and materials}

The datasets generated and analyzed during the current study are not publicly available due to privacy reasons (images of patients), but are available from the corresponding author on reasonable request.

\section{Declarations}

Ethics approval and consent to participate

The study was approved by the Institutional Review Board of the University of Brescia (\#DENMON). Written informed consent was obtained from all participants, and from the parents of those patients younger than 18 years old.

\section{Consent for publication}

Consent for publication was obtained from all patients.

\section{Competing interests}

Dental Monitoring ${ }^{\circledR}$ partially sponsored the cost of the monthly monitoring.

\section{Author details}

${ }^{1}$ Department of Information Engineering, Faculty of Engineering, University of Brescia, Via Branze 38, 25123 Brescia, Italy. ${ }^{2}$ Division of Orofacial Pain, College of Dentistry, University of Kentucky, 740 S. Limestone, Lexington, KY 40536, USA. ${ }^{3}$ Division of Paediatric Dentistry and Orthodontics, Faculty of Dentistry, 2/F, Prince Philip Dental Hospital, The University of Hong Kong, 34 Hospital Road, Sai Ying Pun, Hong Kong SAR. ${ }^{4}$ Dental School, Department of Medical and Surgical Specialties, Radiological Sciences and Public Health, University of Brescia, Piazzale Spedali Civili 1, 25123 Brescia, Italy.
Received: 19 May 2021 Accepted: 27 August 2021

Published online: 07 September 2021

\section{References}

1. Savoldi F, Bonetti S, Dalessandri D, et al. Incisal apical root resorption evaluation after low-friction orthodontic treatment through two-dimensional radiographic imaging and trigonometric correction. J Clin Diagn Res 2015;9:ZC70-ZC4.

2. Al-Anezi SA, Harradine NW. Quantifying plaque during orthodontic treatment: a systematic review. Angle Orthod. 2011;C82:748-53.

3. Lundström F, Krasse B. Streptococcus mutans and lactobacilli frequency in orthodontic patients; the effect of chlorhexidine treatments. EJO. 1987;9:109-16.

4. Geiger AM, Gorelick L, Gwinnett AJ, et al. The effect of a fluoride program on white spot formation during orthodontic treatment. Am J Orthod Dentofac Orthop. 1988;93:29-37.

5. Duijster D, de Jong-Lenters $M$, Verrips $E$, et al. Establishing oral health promoting behaviours in children-parents'views on barriers, facilitators and professional support: a qualitative study. BMC Oral Health. 2015;15:157.s.

6. Zotti F, Pietrobelli A, Malchiodi L, et al. Apps for oral hygiene in children 4 to 7 years: Fun and ef-fectiveness. J Clin Exp Dent. 2019;11:e795-801.

7. Marini I, Bortolotti F, Incerti Parenti S, et al. Combined effects of repeated oral hygiene motivation and type of toothbrush on orthodontic patients. A blind randomized clinical trial. Angle Orthod. 2014;84:896-901.

8. Fjeldsoe BS, Marshall AL, Miller YD. Behavior change interventions delivered by mobile telephone short-message service. Am J Prev Med. c2009;36:165-73.

9. Toniazzo MP, Nodari D, Gomes Muniz FWM, et al. Effect of mHealth in improving oral hygiene; a systematic review with meta-analysis. JCP. 2019;46:297-309.

10. Cozzani M, Ragazzini G, Delucchi A, et al. Oral hygiene compliance in orthodontic patients: a randomized controlled study on the effects of a post-treatment communication. Prog Orthod. 2016;17:17-41.

11. Bowen BT, Rinchuse DJ, Zullo T, et al. The influence of text messaging on oral hygiene effective-ness. Angle Orthod. 2014;85:543-8.

12. Jadhav HC, Dodamani AS, Karibasappa GN, et al. Effect of reinforcement of oral health education message through short messaging service in mobile phones: a quasi-experimental trial. Int J Telemed Appl. 2016;2016:7293516.

13. Zotti F, Dalessandri D, Salgarello S, et al. Usefulness of an app in improving oral hygiene compliance in adolescent orthodontic patients. Angle Orthod. 2016;86:101-7.

14. Harnacke D, Mitter S, Lehner M, et al. Improving oral hygiene skills by computer-based training: a randomized controlled comparison of the modified bass and the fones techniques. PLoS ONE. 2012;7:e37072.

15. Scribante A, Gallo S, Bertino K, Meles S, Gandini P, Sfondrini MF. The effect of chairside verbal instructions matched with instagram social media on oral hygiene of young orthodontic patients: a randomized clinical trial. Appl Sci. 2021;11:706.

16. Guo J, Yan X, Li S, Van der Walt J, Guan G, Mei L. Quantitative and qualitative analyses of orthodontic-related videos on YouTube. Angle Orthod. 2020;90:411-8.

17. Rinchuse DJ, Rinchuse D, Zullo TG. Oral hygiene compliance: a clinical investigation. J Clin Orthod. 1992;26:33-8.

18. Li XXZ, Tang N, et al. Effect of intervention using a messaging app on compliance and duration of treatment in orthodontic patients. Clin Oral Investig. 2016;20:1849-59.

19. Roisin LC, Brezilier D, Sorel O. Remotely-controlled orthodontics: fundamentals and description of the Dental Monitoring system. J Dentofacial Anom Orthod. 2016;19:1-12.

20. Silven AV, Petrus AHJ, Villalobos-Quesada M, et al. Telemonitoring for patients with COVID-19: recommendations for design and implementation. J Med Internet Res. 2020;22:e20953.

21. Pango Madariaga AD, Bucci R, Rongo R, Simeon V, D'Antó V, Valletta R. Impact of fixed orthodontic appliance and clear aligners on the periodontal health: a prospective clinical study. Dent J (Basel). 2020;8:4. 
22. Faul F, Erdfelder E, Lang AG, Buchner A. G*Power 3: a flexible statistical power analysis program for the social, behavioral, and biomedical sciences. Behav Res Meth. 2007;39:175-91.

23. Davies TM, Shaw WC, Worthington HV, et al. The effect of orthodontic treatment on plaque and gingivitis. Am J Orthod Dentofac Orthop. 1991;99:155-61

24. Loe H. The Gingival Index, the Plaque Index and the Retention Index Systems. J Period. 1967;38:610-6.

25. Bianco A, Dalessandri D, Oliva B, Isola G, Tonni I, Bonetti S, et al. COVID-19 and Orthodontics: an approach for monitoring patients at home. The Open Dent J. 2021;15:87-96.

26. Dalessandri D, Sangalli L, Tonni I, Laffranchi L, Bonetti S, Visconti L, et al. Attitude towards telemonitoring in orthodontists and orthodontic patients. Dent J. 2021;9:47

27. Vaid NR. Artificial Intelligence (AI) driven orthodontic care: A quest toward utopia? Semin Orthod. 2021;27:57-61.

28. Hansa I, Katyal V, Semaan SJ, Coyne R, Vaid NR. Artificial intelligence driven remote monitoring of orthodontic patients: clinical applicability and rationale. Semin Orthod. 2021;27:138-56.

29. Pandis N, Vlahopoulos K, Polychronopoulou A, et al. Self-ligating vs conventional brackets in the treatment of mandibular crowding: a prospective clinical trial of treatment duration and dental effects. Am J Orthod Dentofacial Orthop. 2007;132:208-15.

30. Peng $Y, W u R, Q u$ W, et al. Effect of visual method vs plaque disclosure in enhancing oral hygiene in adolescents and young adults: a single-blind randomised controlled trial. Am J Orthod Dentofac Orthop. 2014;145:280-6.

31. Alfuriji S, Alhazmi N, Alhamlan N, et al. The effect of orthodontic therapy on periodontal health: a review of the literature. Int J Dent. 2014;2014:585048.

32. Tasios T, Papageorgiou SN, Papadopoulos MA, et al. Prevention of orthodontic enamel demineraliza-tion: a systematic review with meta-analyses. Orthod Craniofac Res. 2019;22:225-35.

33. Savoldi F, Paganelli C. In vitro evaluation of loop design influencing the sliding of orthodontic wires: a preliminary study. JABFM. 2019;17:1-8.

34. Benson PE, Parking N, Dyer F, et al. Fluorides for preventing early tooth decay (demineralised lesions) during fixed brace treatment. Coch Syst Rev. 2019;12:11.

35. Sedgwick P, Greenwood N. Understanding the Hawthorne effect. BMJ. 2015;351:h4672.

36. Kravitz ND, Burris B, Butler D, et al. Teledentistry, do-it-yourself, orthodontics, and remote treatment monitoring. JCO. 2016;L12:718-25.

37. Hansa I, Katyal V, Ferguson DJ, Vaid N. Outcomes of clear aligner treatment with and without dental monitoring: a retrospective cohort study. Am J Orthod Dentofacial Orthop. 2021;159:453-9.

\section{Publisher's Note}

Springer Nature remains neutral with regard to jurisdictional claims in published maps and institutional affiliations.
Ready to submit your research? Choose BMC and benefit from:

- fast, convenient online submission

- thorough peer review by experienced researchers in your field

- rapid publication on acceptance

- support for research data, including large and complex data types

- gold Open Access which fosters wider collaboration and increased citations

- maximum visibility for your research: over $100 \mathrm{M}$ website views per year

At $\mathrm{BMC}$, research is always in progress.

Learn more biomedcentral.com/submissions 